\title{
Simulation Guided Design of Globular Single-Chain Nanoparticles by Tuning the Solvent Quality
}

\author{
Federica Lo Verso, ${ }^{* a}$ José A. Pomposo, $, c, d, e^{b u a n}$ Colmenero, ${ }^{a, b, c, d}$ and Angel J. Moreno ${ }^{b, c}$
}

Received Xth $X X X X X X X X X X 20 X X$, Accepted Xth $X X X X X X X X X 20 X X$

First published on the web $X t h X X X X X X X X X X 200 X D O I$ : 10.1039/b000000x

\begin{abstract}
The control of primary and further structures of individual folded/collapsed synthetic polymers has received significant attention in recent years. However, the synthesis of single-chain nanoparticles (SCNPs) showing a compact, globular conformation in solution has turned out so far highly elusive. By means of simulations, we propose two methods for obtaining globular SCNPs in solution. The first synthesis route is performed in bad solvent, with the precursor anchored to a surface. In the second route we use a random copolymer precursor with unreactive solvophilic and reactive solvophobic units, which form a single core-shell structure. Both protocols prevent intermolecular cross-linking. After recovering good solvent conditions, the swollen nanoparticles keep their globular character. The proposed methods are experimentally realizable and do not require specific sequence control of the precursors. Our results pave the way to the synthesis via solvent-assisted design of a new generation of globular soft nanoparticles mimicking global conformations of native proteins in solution.
\end{abstract}

\section{Introduction}

Single-chain nanoparticles (SCNPs) are unimolecular soft nano-objects, consisting in individual polymer chains collapsed to a certain degree by means of intramolecular bonding (i.e., covalent, noncovalent or dynamic covalent bonds). ${ }^{1-4}$. SCNPs are promising soft nano-objects for applications in the fields of catalysis, ${ }^{5-8}$ drug delivery, ${ }^{9-12}$ sensing ${ }^{13}$ and bioimaging. ${ }^{14-18}$ It is worth mentioning that SCNPs in solution synthesized by means of state-of-the-art intrachain folding/collapse techniques, show noncompact, nonglobular conformations as revealed by recent small-angle neutron scattering (SANS) and small-angle X-ray scattering (SAXS) measurements, as well as complementary MD simulations. ${ }^{19}$ The underlying physical mechanism for the noncompact morphology of SCNPs in solution has been recently put forward. ${ }^{19-21}$ In brief, the typical extended self-avoiding conformation of the foldable precursor chains, in the good solvent conditions of synthesis, promotes intramolecular bonding to take place mostly between reactive functional groups that are separated by short contour distances. This fact promotes local globulation along the chain, but is not efficient for global, large-scale

\footnotetext{
a Donostia International Physics Center (DIPC), Paseo Manuel de Lardizabal 4, E-20018 San Sebastián, Spain. Tel: +34 943015414; E-mail: federica_loverso001@ehu.es

${ }^{b}$ Centro de Física de Materiales (CSIC, UPV/EHU), Paseo Manuel de Lardizabal 5, E-20018 San Sebastián, Spain.

${ }^{c}$ Materials Physics Center MPC, Paseo Manuel de Lardizabal 5, E-20018 San Sebastián, Spain.

d Departamento de Física de Materiales, Universidad del País Vasco (UPV/EHU), Apartado 1072, E-20080 San Sebastián, Spain.

e IKERBASQUE - Basque Foundation for Science, María Díaz de Haro 3, E-48013 Bilbao Spain.
}

chain compaction. Consequently, the morphology of current SCNPs in solution resembles those observed in intrinsically disordered proteins, having locally compact portions of the chain connected by flexible segments. ${ }^{11,12}$ Even if this has been a major step towards the synthesis of protein-like soft nanoparticles, efficient methods for obtaining globular, compact SCNPs in solution are still lacking.

It may be argued that by performing the SCNP synthesis under bad solvent conditions (i.e., starting from a collapsed precursor), intramolecular bonding between reactive groups separated by long contour distances will be highly favored, eventually leading to globular conformations of the swollen SCNPs after recovering good solvent conditions. However, from an experimental point of view, bad solvent conditions are intrinsically associated to severe polymer insolubility and hence intermolecular aggregation (except for unpractical extreme dilution). This precludes direct SCNP synthesis under such conditions. Consequently, alternative methods are required to construct globular, soft nanoparticles in solution.

This manuscript reports two solvent-assisted design protocols, implemented and validated through molecular dynamics (MD) simulations, for the construction of SCNPs owning globular morphologies in solution. By taking inspiration from Merrifield synthesis using solid supports ${ }^{22}$, we envision a first procedure (model I in Fig. 1) for performing the chain folding/collapse under bad solvent conditions. Namely, foldable precursor chains are anchored to solid supports at sufficiently low surface coverage to avoid intermolecular bonding. Upon individual SCNP formation in bad solvent through intramolecular bonding, the cleavage of the unimolecular particles synthesized in the globular state should be possible, as 
well as their transfer to good solvent conditions in order to determine their structural properties (size and shape). The second procedure is inspired by the general global conformations of native globular proteins. Hence, we hypothesize that decoration of the foldable precursor chains with unreactive solvophilic and reactive solvophobic units may lead to a core-shell conformation that isolates the solvophobic units and prevent intermolecular aggregation. This will allow for SCNP formation via intramolecular bonding of the solvophobic groups. To understand the effect of the distribution of the solvophilic/solvophobic units on the resulting SCNP morphology, random vs. regular sequences of such units along the foldable precursor chain are considered (models II-RAN and II-REG in Fig. 1, respectively).

Also this second route is experimentally realizable. The control of the primary structure of synthetic polymers has indeed received significant attention in recent years, as pioneered by Lutz et al. ${ }^{23}$, and it is envisioned that, working along this line, the control over the secondary and tertiary structure of synthetic polymers will be achieved via singlechain technologies ${ }^{24}$. At the present time two examples of non-covalent bonded SCNPs, constructed by tuning the hydrophilic/hydrophobic sequence and interactions of a random copolymer, have been recently reported ${ }^{25,26}$. In such reported cases the single-chain self-folding is reversible, i.e., the globular conformations are lost when restoring good solvent quality for all monomers. However, irreversible chemical bonding should be easily implemented, producing SCNPs that keep their globular conformation after recovering good solvent conditions.

The manuscript is organized as follows: in section 2 we discuss the models and methods developed in our study. Sections 3 and 4 report about the results obtained via the two different synthesis protocols (model I and II respectively). In section 5 we draw our conclusions.

\section{Model and Methods}

To investigate the feasibility of these new solvent-assisted design protocols (models I and II) for obtaining globular SC$\mathrm{NPs}$, we modified the bead-spring model $^{27}$ used in our previous coarse-grained MD simulations ${ }^{20,21}$ in order to include solvophilic and solvophobic interactions along the precursor chains. Now, the non-bonded pair interaction between beads is given by: ${ }^{28}$

$$
V_{\mathrm{nb}}(r)= \begin{cases}V_{\mathrm{LJ}}(r)=4 \varepsilon\left[\left(\frac{\sigma}{r}\right)^{12}-\left(\frac{\sigma}{r}\right)^{6}+\frac{1}{4}\right]-\varepsilon \phi & r \leq 2^{1 / 6} \sigma \\ V_{\phi}(r)=\frac{1}{2} \phi \varepsilon\left[\cos \left(\alpha\left(\frac{r}{\sigma}\right)^{2}+\beta\right)-1\right] & 2^{1 / 6} \sigma<r \leq 1.5 \sigma \\ 0 & r>1.5 \sigma\end{cases}
$$

By using the parameters ${ }^{28} \alpha=\pi\left(2.25-2^{1 / 3}\right)^{-1}$ and $\beta=$ $2 \pi-2.25 \alpha$, the non-bonded potential and its first derivative are continuous at $r=2^{1 / 6} \sigma$. The quantities $\varepsilon, \sigma$, and $\tau=\left(\sigma^{2} m / \varepsilon\right)^{1 / 2}$ (with $m$ the bead mass) set the energy, length, and time scales, respectively. In what follows we will use Lennard-Jones (LJ) units, $\varepsilon=\sigma=m=\tau=1$. An attractive tail, acting on a distance corresponding to the first neighbor shell $\left(2^{1 / 6} \leq r \leq 1.5\right)$, can be switched-on and off by changing the depth $\phi$. For $\phi=0$ the purely repulsive LJ, excluded volume interaction is recovered, which represents the pair interaction among solvophilic groups. By increasing $\phi$ the interaction between the solvophobic units can be tuned, eventually inducing their collapsed state. All simulations were performed at temperature $T=1$. To guarantee collapse at that $T$, we used a value $\phi=2$ far below the $\Theta$-point. Indeed, by analyzing the derivative of the gyration radius of the precursor vs. $\phi$, for $T=1$ the $\Theta$-point was estimated to occur at $\phi \sim 0.7$. Permanent bonding between two connected beads is introduced through a FENE (finitely extensible non-linear elastic) poten- tial $^{27}$,

$$
V_{\mathrm{FENE}}(r)=-\varepsilon K_{\mathrm{F}} R_{0}^{2} \ln \left[1-\left(\frac{r}{R_{0} \sigma}\right)^{2}\right],
$$

with $K_{\mathrm{F}}=30$ and $R_{0}=1.5$. In order to stabilize the temperature $T=1$ of the system, we implement a Langevin thermostat. For further simulation details see Refs. ${ }^{20,21}$.

We have investigated three different synthesis routes corresponding to different models for the chemical composition of the precursor. Fig. 1 shows a schematic representation of the investigated models. For the model I the architecture 20,21 consists of a linear backbone of $N$ beads, each of them being attached to a side group. There are two kinds of side groups, which are randomly distributed along the backbone. A number $N_{1}$ of the side groups contain three beads. The free end beads (green beads in Fig. 1) are the 'reactive' functional groups that can form mutual bonds. The remaining $N-N_{1}$ side groups contain a single unreactive bead. The fraction of functional groups is defined as $f=N_{\mathrm{l}} / N$. This model has been used in the study of the nanoparticle synthesis in bad solvent condi- 


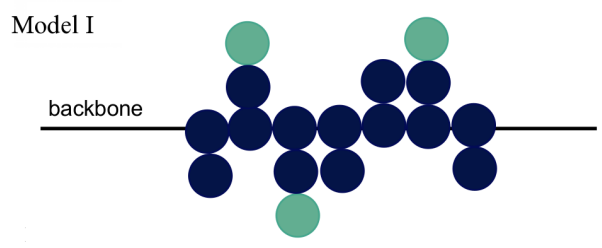

Model II-RAN

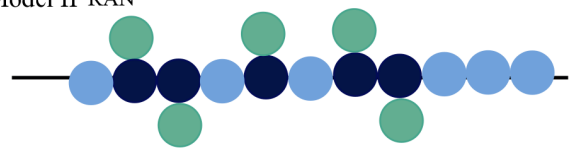

Model II-REG

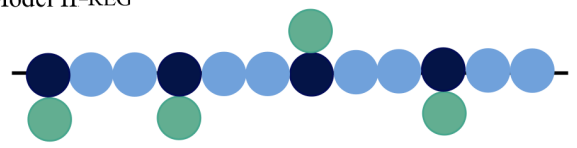

Fig. 1 Schematic representation of the polymer precursor for the three investigated models. Green beads are the reactive functional groups (solvophobic in all cases). Dark-blue and light-blue beads are the unreactive solvophobic and solvophilic units, respectively. In models II-RAN and II-REG the sequence of solvophobic and solvophilic units is random and regular, respectively.

tions, i.e., all the monomers are solvophobic during the crosslinking process. As mentioned above, real synthesis cannot be performed at arbitrarely high dilution. Thus, in practice, if the nanoparticle synthesis is performed at bad solvent conditions the system unavoidingly undergoes aggregation and intermolecular cross-linking. However, this propensity can be prevented by bonding the individual precursors to a surface, prior to lowering the solvent quality below the $\Theta$-point and initiating cross-linking. If the density of polymers in the surface is suficiently low, anchoring to the surface avoids contact between different polymers and intermolecular aggregation, irrespective of the solvent quality. To model a non-penetrable surface, a purely repulsive external LJ-potential, $V_{\mathrm{s}}(z)$, has been imposed to all beads in the system of model I:

$$
V_{\mathrm{s}}(r)= \begin{cases}4 \varepsilon\left[\left(\frac{\sigma}{z}\right)^{12}-\left(\frac{\sigma}{z}\right)^{6}+\frac{1}{4}\right] & z \leq 2^{1 / 6} \sigma \\ 0 & z>2^{1 / 6} \sigma\end{cases}
$$

with $z$ the normal distance to the plane $z=0$. One of the end beads of the backbone is linked to a fixed point of the plane $z=0$, through the same FENE potential of Eq. 2 . The whole precursor is initially placed at the side $z>0$, so that the potential $V_{\mathrm{s}}(z)$ prevents penetration $(z<0)$ of the surface.

The system of models II-RAN and II-REG is a copolymer containing two kind of units: a single unreactive solvophilic bead, and a second unit formed by two solvophobic beads. One of the solvophobic beads is unreactive, whereas the other is a reactive functional side group, directly attached to the main backbone. Thus, the backbone is formed by all the unreactive (solvophobic or solvophilic) groups (see Fig. 1). In the model II-RAN the solvophobic and solvophilic units are randomly distributed along the copolymer. A regular distribution is instead used in the model II-REG, where the repeating basic pattern is just dictated by the value of $f$. The crossinteraction between solvophilic and solvophobic units is in all cases purely repulsive $(\phi=0)$.

The reactive groups in the three models are monofunctional, i.e, they can form a single bond with other functional groups within the same chain. Thus, a new bond between two functional groups is formed if two conditions are fulfilled: i) none of them are bonded to other functional group, and ii) they are at a mutual distance $r<1.3 \sigma$ ('capture distance'). A random choice is made in case of multiple options (several functional groups within the same capture distance). When a new bond is formed, the two involved beads interact through the FENE potential of Eq. 2. We have to stress that cross-linking is irreversible, i.e, when a new bond is formed, this is permanent (the FENE interaction is switched on for the rest of the simulation). However, the self-assembling of the solvophobic groups is reversible. Consequently the nanoparticles can be swollen by restoring the purely repulsive LJ potential $(\phi=0)$ for the non-bonded interaction among all monomers, mimicking good solvent conditions for all species.

The simulation protocol has three steps. First the precursor is equilibrated, with the attractive tail of the non-bonded potential switched on $(\phi=2)$ for all the solvophobic beads (all the beads in the case of model I). Thus, the precursor adopts a collapsed conformation, which in models II-RAN and II-REG corresponds to a core-shell structure where the solvophobic units are isolated by the solvophilic ones (see below). In the second step the cross-linking is activated by allowing the functional groups to form mutual bonds. Finally, after completing the cross-linking, good solvent conditions are imposed for all beads by switching off the attractive interactions $(\phi=0)$, and the nanoparticle adopts a swollen conformation. In model I the nanoparticle is moreover cleaved from the surface by removing the potential of Eq. 3. In these conditions an acquisition run is performed for analysis of the nanoparticle structure obtained from each model. For each value of $N$ and $f$ at least 100 independent trajectories are simulated.

For the model I we have explored precursor lengths $25 \leq$ $N \leq 1600$ and a broad range of $f$-values $(0.04 \leq f \leq 0.72)$. For the models II-RAN and II-REG we have explored lengths $44 \leq N \leq 414$ at fixed $f=0.30$. Larger values of $f$ in the amphiphilic systems are not useful in practice, since the solvophilic units are not sufficient to fully isolate the solvophobic core from the solvent. On the other hand, small fractions $(f \lesssim 0.2)$ do not lead to a single core-shell structure except for very small $N$. Instead, the solvophobic units aggregate into separate cores and the precursor forms a sparse chain 
of connected globules, each globule containing a single coreshell. Cross-liking of these multi-core systems does not yield globular nanoparticles and will be discussed elsewhere.

\section{Results and discussion: model I}

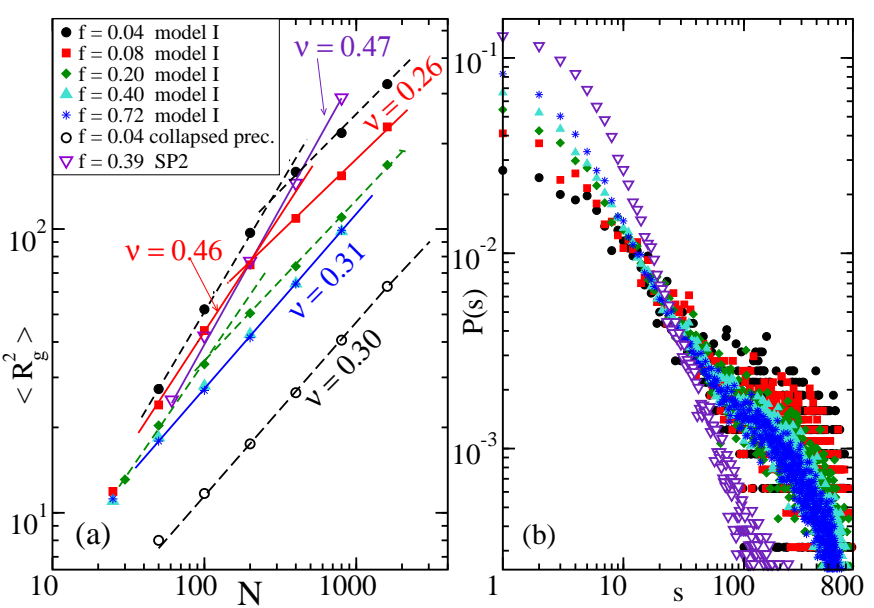

Fig. 2 Model I (swollen nanoparticles): a) Average squared radius of gyration vs. the backbone length $N$ for all the investigated fractions $f$ of functional groups. We include results $(f=0.04$, open circles) for the collapsed unlinked precursor (i.e., in bad solvent and prior to initiating cross-linking), as well as for SCNPs of model SP2 with $f=0.39$ (inverted triangles) $^{20,21}$. Symbols are simulation data. Solid and dashed lines are fits to power-laws $R_{g}^{2} \sim N^{2 v}$ (the representative exponents are indicated). b) Normalized histogram of contour distances $s$ between the bonded functional groups of the nanoparticle, for $N=800$ and several $f$-values.

Fig. 2a shows results for the $N$-dependence of the average squared radius of gyration, $\left\langle R_{\mathrm{g}}^{2}\right\rangle$, of the swollen nanoparticles of model I. Data are represented for all the investigated range of $f$. The symbols correspond to the MD data. The lines are fits of the data to the scaling law ${ }^{29}\left\langle R_{\mathrm{g}}^{2}\right\rangle \sim N^{2 v}$. We include representative results $(f=0.04)$ for the collapsed precursor (i.e., in bad solvent and prior to initiating cross-linking), which as expected shows the exponent $v \approx 1 / 3$ characteristic of globular objects. ${ }^{29}$ For comparison we also include results for the SCNPs of the model SP2 (case $f=0.39$ ) of Refs. ${ }^{20,21}$ In the model SP2 cross-linking is performed starting from the same precursor of model I, but in good solvent conditions $(\phi=0)$ for all beads. Moreover there are two different types of functional groups (in identical proportion) and only bonding between groups of the same type are permitted. This model mimics synthesis of single-chain nanoparticles by using orthogonal chemistry, which leads to more compact objects than their counterparts with a single type of functional groups, though these are still far from globular conformations. ${ }^{20,21}$
The scaling exponent for the model SP2 is $v \approx 1 / 2$, reflecting gaussian-like conformations, ${ }^{29}$ and is essentially independent of $f .{ }^{20,21}$ Results in Fig. 2a demonstrate the efficiency of the cross-linking route in bad solvent: even with a very small fraction of functional groups, a considerable reduction of the exponent $v$ for the swollen nanoparticles can be obtained compared to those synthesized in good solvent conditions. This is due to the efficiency of the used protocol in increasing the probability of bonding at long contour distance, with respect to the good solvent case. The contour distance, $s=|i-j|$ is defined as the number of backbone beads comprised between the two backbone beads $(i, j)$ to which the side groups of the mutually bonded functional groups are attached. ${ }^{20,21}$ In Fig. 2b, we show the distribution $P(s)$ of the contour distance between bonded functional groups in the nanoparticle, for different $f$-values at fixed $N=800$. A clear increase, by more than one order of magnitude with respect to nanoparticles synthesized in good solvent, is found at large $s$ values. Because of the highly folded conformation of the backbone in the globular state, the probability of contact between two functional groups is weakly dependent on their contour distance, except in the limits $s \rightarrow 1$ and $s \rightarrow N$. As a consequence, $P(s)$ for the nanoparticles synthesized in bad solvent forms a plateau at $1 \ll s \ll N$ (Fig. 2b). In the extreme limits, $s \rightarrow 1$ and $s \rightarrow N$, the probabilities are trivially high and low, respectively, for all the solvent conditions.

A detailed analysis of the scaling behavior of $\left\langle R_{\mathrm{g}}^{2}\right\rangle$ in Fig. 2a reveals interesting features. A single scaling exponent $v \approx 1 / 3$ (as expected for globular objects), is observed for high fraction of functional groups $f \geq 0.4$. This value confirms that for high enough $f$, the swollen nanoparticles have globular conformations, as opposite to the open sparse conformations $(v \approx 1 / 2$ irrespective of $f$ ) observed for nanoparticles synthesized in good solvent. ${ }^{20,21}$ In contrast to the good solvent case ${ }^{20}$, a strong and complex dependence on $f$ emerges for the scaling behavior of the nanoparticles of model I. Thus, for $f \leq 0.2$ two different scaling exponents are found, which depend on the length of the backbone. In particular for small precursors, by decreasing $f$ we observe a progressive increase of $v$, approaching the exponent $v \approx 1 / 2$ that is obtained for nanoparticles synthesized in good solvent. Indeed, independently from the quality of the solvent, for small values of both $N$ and $f$ the number of bonds in the fully cross-linked nanoparticle is too low to form globular objects (e.g., just two bonds for $N=100$ and $f=0.04$ ). However, in bad solvent conditions, there is a significant advantage connected to the possibility of tuning the nanoparticle structure by changing $f$. For cross-linking in bad solvent the increase of $f$ does provide an efficient route for creating long-range loops, and the expected exponent $v=1 / 3$ is found for $f>0.2$. On the other hand, in good solvent the growth of $f$ mostly produces bonds at short contour distances that are inefficient for global compaction ${ }^{20,21}$, and $v$ shows 

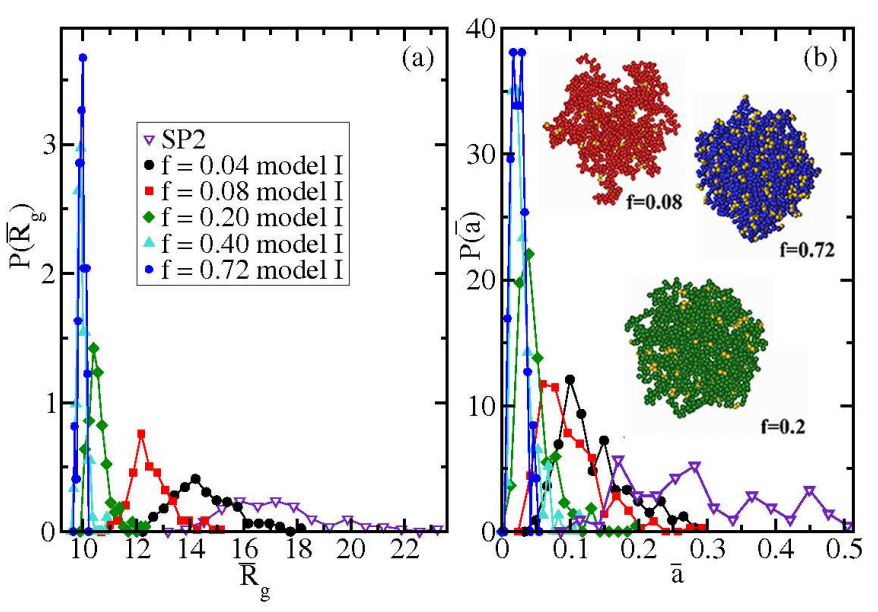

Fig. 3 Distributions of the time-averaged radius of gyration and asphericity for $N=800$ and several $f$-values, obtained for the swollen nanoparticles of model I (typical snapshots shown in (b)). For comparison we include the distributions for the model SP2 with $N=800$ and $f=0.39 .^{21}$

just minor changes with $f$.

For long precursors, the opposite behavior is observed: decreasing $f$ yields lower values of $v$. Thus, for the lowest investigated fractions of functional groups, $f=0.04$ and $f=0.08$, large nanoparticles scale with exponent $v \approx 1 / 4$. This scaling behavior at very low $f$ resembles that obtained for vulcanized networks ( $v=1 / 4$ in mean-field approach), ${ }^{29}$ which are obtained by cross-linking of strongly overlapped long linear chains with a very low fraction $(f \ll 1)$ of reactive monomers per chain. The precursor lenght for the crossover between the small and large- $N$ regimes of $\left\langle R_{\mathrm{g}}^{2}\right\rangle$ depends on the fraction of functional groups, increasing from $N \sim 100$ at $f=0.2$ to $N \sim 300$ at $f=0.04$. This is consistent with the fact that, as discussed above, a critical number of bonds is needed for efficient compaction of the fully cross-linked nanoparticle. Thus, for lower $f$ that critical number will arise at larger $N$.

In Fig. 3 we show typical snapshots of the obtained swollen nanoparticles of model I, for fixed $N=800$ and different fractions of funtional groups. Nanoparticles with low $f$ are less compact and spherical, and present more protrusions than the densely cross-linked nanoparticles obtained for high $f$. As discussed in Refs. ${ }^{20,21}$, irreversible cross-linking from different initial realizations of a same precursor leads to topologically polydisperse nanoparticles (different connectivity in the network of bonds). This feature can be quantified by computing the distributions of the time-averaged radius of gyration $\bar{R}_{\mathrm{g}}$ and asphericity parameter $\bar{a}$. The latter quantifies deviations from a sphero-symmetrical shape $(a=0)$. The timeaveraged values of $R_{\mathrm{g}}$ and $a$ for an individual swollen nanoparticle are just obtained by averaging their corresponding instan-
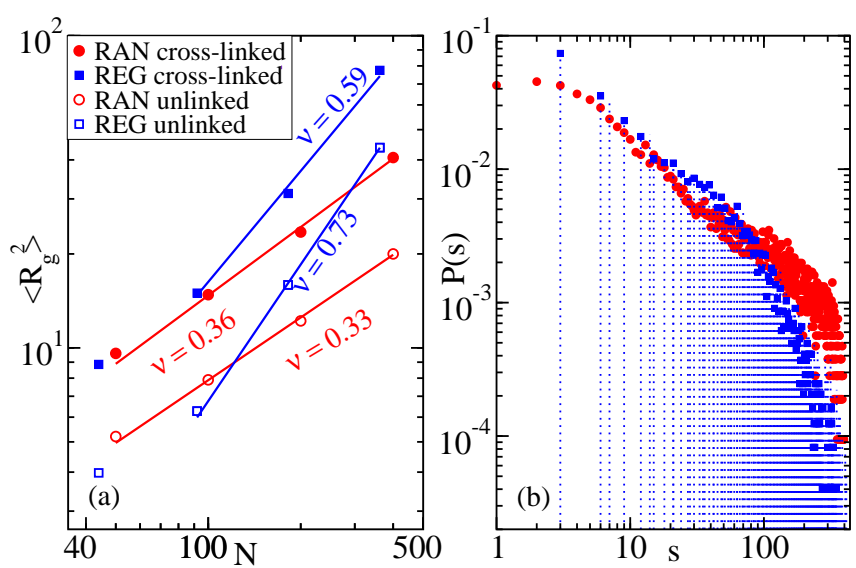

Fig. 4 Amphiphilic systems (models II): a) Results for the average squared radius of gyration vs. the backbone length $N$. Full symbols are simulation data for the swollen nanoparticles of model II-RAN (circles) and II-REG (squares), i.e., obtained from precursors with random and periodic composition pattern, respectively. We include results (open symbols) for the collapsed unlinked precursors (i.e., in bad solvent and prior to initiating cross-linking). Lines are fits to power-laws $\left\langle R_{\mathrm{g}}^{2}\right\rangle \sim N^{2 v}$ (exponents indicated). b) Histogram of contour distances $s$ between the bonded functional groups of the nanoparticles with $N=400$. Because of the specific periodic distribution of the reactive groups along the REG-precursor, bonds are only formed for integer multiples of $s=3$, as indicated by the vertical dotted lines. As a consequence the amplitude of the normalized $P(s)$ for the REG-system is much higher than for the RAN-system. To facilitate the comparison, $P(s)$ for the REG-system has been scaled by $1 / 3$.

taneous values over the acquisition run. ${ }^{20,21}$ Fig. 3 shows the distributions $P\left(\bar{R}_{\mathrm{g}}\right)$ and $P(\bar{a})$ for fixed $N=800$ and different values of $f$. For comparison we include the corresponding results for the model SP2 with $f=0.39$. The results in Fig. 3 confirm that, even for very small $f=0.04$, the swollen nanoparticles synthesized in bad solvent are much less sparse and much closer to the spherical shape than their counterparts synthesized in good solvent with high $f$. By increasing $f$ in the model I, nearly spherical conformations are obtained for $f \geq 0.4$, as shown by the narrow distributions $P(\bar{a})$ centered at $\bar{a} \sim 0.02$.

\section{Results and discussion: model II}

Now we discuss the results found for the nanoparticles obtained from the amphiphilic precursors of models II-RAN and II-REG, i.e., with random (RAN) and regular (REG) composition pattern, respectively. Fig. 4 a shows the $N$-dependence of the average squared radius of gyration. Whereas the swollen nanoparticles of both systems have a very similar size for $N \leq 100$, for longer precursors they display a very different 
behavior. This can be effectively described by a power-law $\left\langle R_{\mathrm{g}}^{2}\right\rangle \sim N^{2 v}$. As obtained in model I, for the RAN-system we find the exponent $v \approx 1 / 3$ expected for a globular object. On the contrary, for the REG-system the data can be described with the Flory exponent $v=0.59$, characteristic of self-avoiding chains. ${ }^{29}$ These rather different features for the RAN- and REG-systems can be understood by inspection of the conformations (Figure 5) and scaling behavior (Fig. 4a) of the respective collapsed precursors. For small lengths $(N \leq 100)$ the collapsed precursor adopts a nearly spherical core-shell conformation, irrespective of the character (random or regular) of the composition pattern. In the case of the RAN-system, the nearly spherical core-shell conformation of the collapsed precursor is found over the whole $N$-range (Figure 5$)$. This is confirmed by its scaling behavior $(v \approx 1 / 3$, open circles in Fig. 4a). In this situation crosslinking of the solvophobic groups within the core has analogies with the model I, and the nanoparticles of model II-RAN keep their globular conformation after swelling. This is consistent with the exponent observed for the swollen nanoparticles $v \approx 1 / 3$ (full circles in Fig. 4a). The situation for long precursors $(N>100)$ in the REG-system is rather different. Though the equilibrium conformation of the precursor in the collapsed state is still a single solvophobic core surrounded by a solvophilic shell, its shape at long $N$ is far from spherical. Instead, due to the regular sequence of the solvophilic and sovophobic groups, an elongated tubular conformation is formed (Figure 5). Indeed the scaling behavior of the collapsed precursor (Fig. $4 \mathrm{a}$ ), shows an exponent $v \approx 0.73$ intermediate between that expected for self-avoiding chains $(v=0.59)$ and rigid rods $(v=1)$. In such a situation cross-linking between solvophobic groups separated by large contour distances will be strongly unfavored, in close analogy with the synthesis in good solvent conditions. This is consistent with the much steeper decay of $P(s)$ at long $s$ in the REG-system than in the RAN-system (see comparison in Fig. $4 \mathrm{~b}$ for $N=400$ ).

As expected from the observed scaling behavior of $\left\langle R_{\mathrm{g}}^{2}\right\rangle$ at $N>100$, the swollen nanoparticles obtained from the RANand REG-systems are globular and sparse, respectively; see representative snapshots for $N=400$ in Fig. 6. This is confirmed by computing the corresponding distributions of timeaveraged radii of gyration and asphericities (see Fig. 6). The swollen nanoparticles of the RAN-system are obviously much smaller and spherical than those of the REG-system. This amazing result opens new ways to obtaining globular singlechain nanoparticles without precise sequence-control. Still, this method is less sucessful than the route of model I (synthesis in pure bad solvent). Indeed with just a $4 \%$ of functional groups the asphericities of the nanoparticles of model I are similar to those of the RAN-system with $f=0.30$ (both distributions are peaked at $\bar{a} \sim 0.1$, see Fig. $3 \mathrm{~b}$ and Fig. 6b). On the other hand, from an experimental point of view, the imple- mentation of method II-RAN is easier than that of method I (this requiring surface anchoring and cleavage steps).
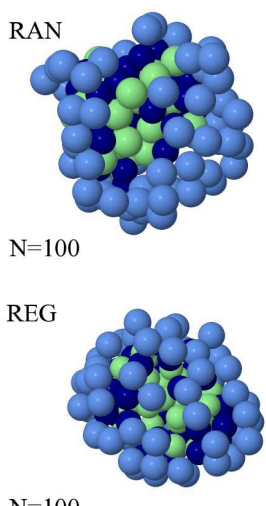

$\mathrm{N}=100$
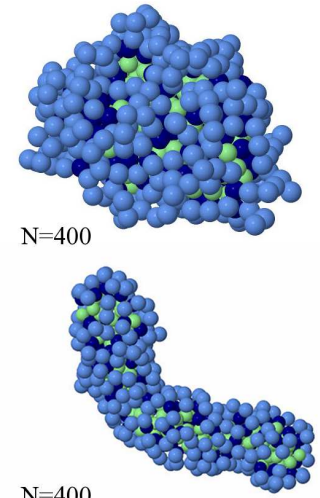

$\mathrm{N}=400$
Fig. 5 Snapshots of equilibrated precursors in bad solvent, prior cross-linking: Model II-RAN (top) and Model II-REG (bottom), for fixed $f=0.3$ and two different lengths of the backbone.

It is worth noting that the observed exponent, $v \approx 0.59$, for the swollen nanoparticles of the model II-REG is higher than that found for synthesis in good solvent $(v \approx 1 / 2) .{ }^{20,21}$ It seems that cross-linking within the solvophobic core, in addition to the regular distribution of the reactive groups, creates a locally tight network of bonds. This might induce an effective local stiffness in the swollen nanoparticles favoring expanded coils $(v \approx 0.59)$ over the gaussian-like $(v \approx 0.5)$ conformations that are obtained by synthesis in good solvent. Whether the scaling with $v \approx 0.59$ in the REG-system extends to the large- $N$ limit or is an intermediate regime, prior to an ultimate crossover to $v=1 / 2$, is an open issue. Indeed the computational cost necessary to reach the equilibrium core-shell structure for longer precursors $\left(N>10^{3}\right)$ is considerably higher than for the systems investigated here.

In the attempt to further highlight the differences in the particular globular conformations of the swollen nanoparticles, while changing the synthesis protocol, we calculate their scattering form factors, $w(q) .{ }^{29}$ Data of $w(q)$ are represented in Fig. 7 for all the investigated cases of Models I and II-RAN. For a fair comparison between the different data sets, $w(q)$ is normalized by $w(q=0)=N_{\text {mon }}$, i.e, by the number of monomers of the nanoparticle, and wavevectors are scaled by the respective radius of gyration. As a general feature, after the Guinier regime which corresponds to $q R_{\mathrm{g}}<1,{ }^{29}$ we observe a sharp drop of the curve, typical of soft colloids with a global spherical structure (e.g., microgels, high functionality stars, spherical brushes/micelles). ${ }^{30-33}$ Thereafter the behavior of the nanoparticles synthesized from Model I depends on the fraction $f$ of functional groups. For small $f$, we find a regime qualitatively similar to the one observed for 

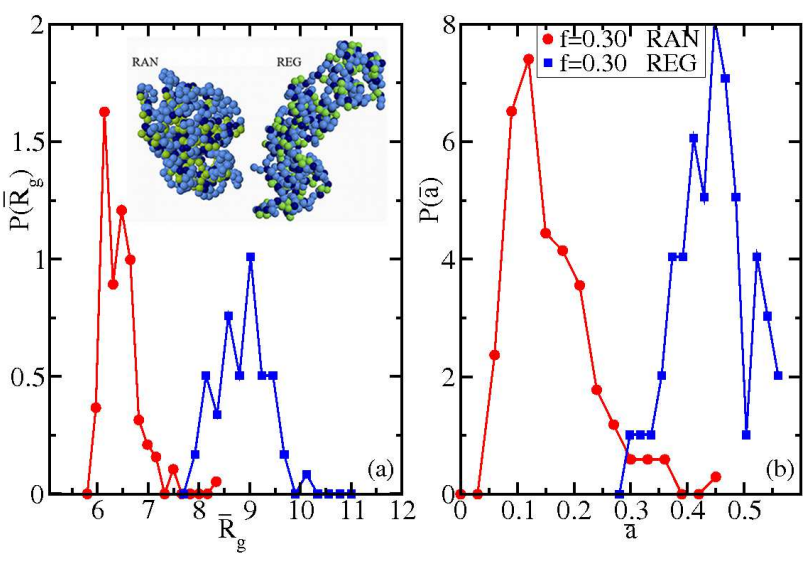

Fig. 6 Amphiphilic systems (models II): for the swollen nanoparticles, (a) distributions of the time-averaged radius of gyration (a) and asphericity (b). Circles and squares correspond to the RAN- and REG-models, respectively. All results correspond to the case $N=400$ and $f=0.30$. The snapshots in (a) correspond to characteristic configurations of the swollen nanoparticles in both models.

isolated polymer chains in good solvent, where the form factor scales as $q^{-1 / v_{\mathrm{F}}}$, with $v_{\mathrm{F}}=0.59$ the Flory exponent. ${ }^{29}$ For small $f$, the swollen nanoparticle contains very long linear segments between cross-linking points. Such long segments can be seen, at intermediate length scales, as diluted chains, leading to the observed scaling in $w(q)$. Instead, by increasing the number of functional groups, and consequently the compactness of the related nanoparticles, an oscillatory behavior emerges, which is reminiscent of the one expected for hard-spheres. ${ }^{30}$ Here the oscillations quickly damp as a consequence of the intrinsic deformability of the soft nanoparticles. A similar trend is observed for soft colloids with high monomer concentration in the interior. ${ }^{30,32,33}$ Finally, at $q R_{\mathrm{g}}>50$ we observe the peak corresponding to the nearest-neighbour monomer correlation $(2 \pi / q \approx \sigma)$, followed by the higher- $q$ harmonics. At low and intermediate $q$-values $\left(q R_{\mathrm{g}}<15\right)$, the curve observed for Model II-RAN and $f=0.30$ is very similar to the one obtained for Model I at a lower fraction of functional groups, $f=0.20$. The difference between both curves at higher $q$ is related to the different local architecture of the respective models (longer side groups in Model I, see Fig. 1).

\section{Conclusions}

In summary, by using computer simulations we have proposed two new routes for the design of globular single-chain nanoparticles, an issue that has remained elusive with usual synthesis protocols, which instead lead to open sparse objects. ${ }^{19-21}$ The two new routes proposed here are based on

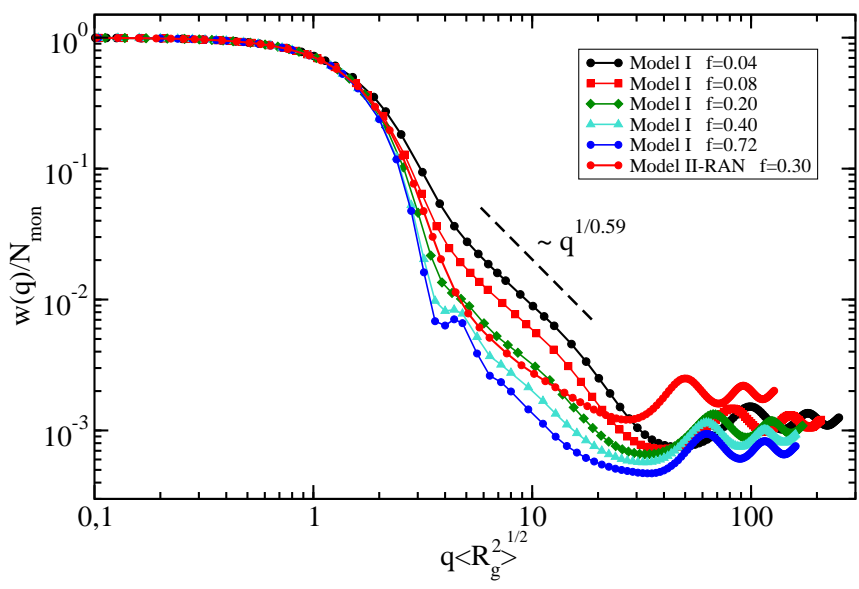

Fig. 7 Scattering form factor calculated for Model I (several $f$ values) and Model II-RAN ( $f=0.30)$. In all cases $N=400$.

tuning the solvent quality, are experimentally realizable with state-of-the-art methods, and do not require specific sequence control. In the first route (method I) cross-linking is performed in bad solvent conditions with the precursors anchored to a surface, at a density sufficiently low to prevent interchain contact. After completing the cross-linking, the nanoparticles are cleaved from the surface. In the second route (method II) a random copolymer with unreactive solvophilic and reactice solvophobic monomers is used, tuning the fraction of both monomers in order to form a single core-shell structure. The solvophilic shell prevents intermolecular aggregation, and the cross-linking of the solvophobic units is purely intramolecular. In both routes, after completing cross-linking and restoring good solvent conditions, the swollen single-chain nanoparticles are globular objects, as confirmed by the analysis of their scaling behavior and asphericity parameter. It is worth noting that amphiphiles with regular sequence are not useful for producing globular nanoparticles, unlike their (easier to synthesize) counterparts with random sequence. Although the crosslinking protocol based on amphiphiles with random composition is less sucessful than the route of the method I, from an experimental point of view, the method II should be more easily implemented. To conclude, the two solvent-assisted design protocols reported in this manuscript and validated by MD simulations pave the way to the synthesis of real globular soft nanoparticles, mimicking the global conformation of globular native proteins in solution for their use in catalysis and nanomedicine, among other potential uses.

We acknowledge financial support from the Projects MAT2012-31088 (MINECO) and T-654-13 (GV), and generous allocation of CPU time from CSUC (Spain). 


\section{References}

1 O. Altintas and C. Barner-Kowollik, Macromol. Rapid Commun., 2012, 33, 958-971.

2 A. Sanchez-Sanchez, I. Perez-Baena and J. A. Pomposo, Molecules, 2013, 18, 3339-3355.

3 A. Sanchez-Sanchez and J. A. Pomposo, Part. Part. Syst. Charact., 2014, 31, 11-23.

4 J. A. Pomposo, Polym. Int., 2014, 63, 589-592.

5 T. Terashima, T. Mes, T. F. A. De Greef, M. A. J. Gillissen, P. Besenius, A. R. A. Palmans and E. W. Meijer, J. Am. Chem. Soc., 2011, 133, 4742 4745.

6 I. Perez-Baena, F. Barroso-Bujans, U. Gasser, A. Arbe, A. J. Moreno, J. Colmenero and J. A. Pomposo, ACS Macro Lett., 2013, 2, 775-779.

7 E. Huerta, P. J. M. Stals, E. W. Meijer and A. R. A. Palmans, Angew. Chem. Int. Ed., 2013, 52, 2906-2910.

8 A. Sanchez-Sanchez, A. Arbe, J. Colmenero and J. A. Pomposo, ACS Macro Lett., 2014, 3, 439-443.

9 S. K. Hamilton and E. Harth, ACS Nano, 2009, 3, 402410.

10 G. Njikang, G. Liu and L. Hong, Langmuir, 2011, 27, 7176-7184.

11 A. Sanchez-Sanchez, S. Akbari, A. Etxeberria, A. Arbe, U. Gasser, A. J. Moreno, J. Colmenero and J. A. Pomposo, ACS Macro Lett., 2013, 2 491-495.

12 A. Sanchez-Sanchez, S. Akbari, A. J. Moreno, F. Lo Verso, A. Arbe, J. Colmenero and J. A. Pomposo, Macromol. Rapid Commun., 2013, 34, 1681-1686.

13 M. A. J. Gillissen, I. K. Voets, E. W. Meijer and A. R. A. Palmans, Polym. Chem., 2012, 3, 3166-3174.

14 L. Oria, R. Aguado, J. A. Pomposo and J. Colmenero, Adv. Mater., 2010, 22, 3038-3041.

15 I. Perez-Baena, I. Loinaz, D. Padro, I. Garcia, H. J. Grande and I. Odriozola, J. Mater. Chem., 2010, 20, 6916-6922.

16 G. Qian, B. Zhu, Y. Wang, S. Deng and A. Hu, Macromol. Rapid Commun., 2012, 33, 1393-1398.

17 J. Willenbacher, K. N. R. Wuest, J. O. Mueller, M. Kaupp, H.-A. Wagenknecht and C. Barner-Kowollik, ACS Macro Lett., 2014, 3, 574-579.

18 D. Chao, X. Jia, B. Tuten, C. Wang and E. B. Berda, Chem. Commun., 2013, 49, 4178-4180.

19 J. A. Pomposo, I. Pérez-Baena, F. Lo Verso, A. J. Moreno, A. Arbe and J. Colmenero, ACS Macro Lett., 2014, 3, 767-772.

20 A. J. Moreno, F. Lo Verso, A. Sanchez-Sanchez, A. Arbe, J. Colmenero and J. A. Pomposo, Macromolecules, 2013, 46, 9748-9759.

21 F. Lo Verso, J. A. Pomposo, J. Colmenero and A. J. Moreno, Soft Matter, 2014, 10, 4813-4821.

22 R. B. Merrifield, J. Am. Chem. Soc., 1963, 85, 2149-2154.

23 J.-F. Lutz, M. Ouchi, D. R. Liu and M. Sawamoto, Science, 2013, 341, 628.

24 M. Ouchi, N. Badi, J.-F. Lutz and M. Sawamoto, Nature Chem., 2011, 3, 917-924.

25 P. Piyapakorn, T. Akagi, M. Hachisuka, T. Onishi, H. Matsuoka and M. Akashi, Macromolecules, 2013, 46, 6187-6194.

26 T. Terashima, T. Sugita, K. Fukae and M. Sawamoto, Macromolecules, 2014, 47, 589-600.

27 K. Kremer and G. S. Grest, J. Chem. Phys., 1990, 92, 5057.

28 T. Soddemann, B. Dünweg and K. Kremer, Eur. Phys. J. E, 2001, 6, 409419.

29 M. Rubinstein and R. H. Colby, Polymer Physics, Oxford University Press, Oxford, UK, 2003.

30 M. Stieger, W. Richtering, J. S. Pedersen and P. Lindner, J. Chem. Phys, 2004, 120, 6197-6206.

31 L. Willner, O. Jucknischke, D. Richter, J. Roovers, L.-L. Zhou, P. M Toporowski, L. J. Fetters, J. S. Huang, M. Y. Lin and N. Hadjichristidis,
Macromolecules, 1994, 27, 3821-3829.

32 F. Lo Verso, S. A. Egorov, A. Milchev and K. Binder, J. Chem. Phys., 2010, 133, 184901

33 S. Förster, E. Wenz and P. Lindner, Phys. Rev. Lett., 1996, 77, 95-98. 


\section{For Table of Contents Use Only}

Typical configurations of swollen single-chain nanoparticles, obtained by two different routes. These are based on intramolecular cross-linking under bad solvent conditions for the reactive groups. Good solvent conditions (leading to nanoparticle swelling) are restored after completing cross-linking. Green beads are the solvophobic reactive groups. The other beads are the unreactive groups: solvophobic (dark blue) and solvophilic (light blue).

Authors: Federica Lo Verso, José A. Pomposo, Juan Colmenero, and Angel J. Moreno
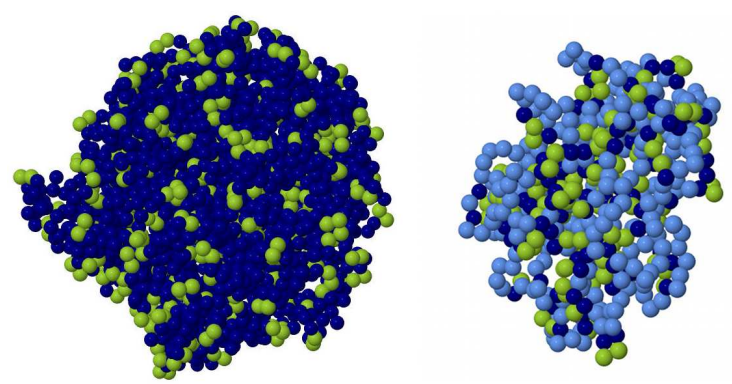

TOC graph 